\title{
Search for Muonic Dark Forces at BABAR
}

\author{
Romulus Godang ${ }^{1, a}$ \\ On Behalf of the BABAR Collaboration \\ ${ }^{1}$ Department of Physics \\ University of South Alabama \\ 411 University Boulevard, North \\ Mobile, AL 36688
}

\begin{abstract}
Many models of physics beyond Standard Model predict the existence of light Higgs states, dark photons, and new gauge bosons mediating interactions between dark sectors and the Standard Model. Using a full data sample collected with the BABAR detector at the PEP-II $e^{+} e^{-}$collider, we report searches for a light non-Standard Model Higgs boson, dark photon, and a new muonic dark force mediated by a gauge boson $\left(Z^{\prime}\right)$ coupling only to the second and third lepton families. Our results significantly improve upon the current bounds and further constrain the remaining region of the allowed parameter space.
\end{abstract}

\section{Introduction}

Many astrophysical and cosmological observations indicate that a fraction of energy density in the universe is due to non-baryonic matter. The microscopic nature of dark matter is currently unknown. Models of physics beyond Standard Model predict the existence of a new non-Abelian gauge group Higgs with gauge boson masses below $10 \mathrm{GeV}$ [1]. The WIMP hypothesis suggested that dark matter is assumed to consist of stable particle with low masses. Such new gauge bosons can typically interact with other Standard Model elementary particles. The new gauge boson, $Z^{\prime}$, can couple to the Standard Model field. SM fields can be directly charged under new gauge boson or the $Z^{\prime}$ may couple with the SM hypercharge boson [2].

Based on the $L_{\mu}-L_{\tau}$ model [3] one of the most promising candidates based on gauging the existing approximate global symmetries of the Standard Model (SM) is the gauge group associated with the difference between muon and tau-lepton number. The gauge $L_{\mu}-L_{\tau}$ model portal to the $Z^{\prime}$ has all features of being coupled only to the leptons of the second and third generation. The $L_{\mu}-L_{\tau}$ model has been studied in the neutrino mass model. It has also been studied in the (g-2) current discrepancy. Consequently it was found that the tentative explanation of the (g-2) anomaly in models with large electron coupling is now excluded at least for multi-GeV and heavier $Z^{\prime}$ bosons. The model explains the possible production of $Z^{\prime}$ gauge boson production via $e^{+} e^{-} \rightarrow \rightarrow \mu^{+} \mu^{-} Z^{\prime}, Z^{\prime} \rightarrow \mu^{+} \mu^{-}$ that primarily comes from the radiation of heavy-flavor leptons and is shown in the following Fig. 1.

\footnotetext{
a e-mail: godang@southalabama.edu
} 


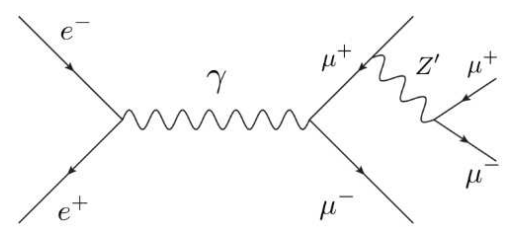

Figure 1. Feynman Diagram for production of gauge boson $Z^{\prime}$ based on the $L_{\mu}-L_{\tau}$ model at an $e^{+} e^{-}$collider.

\section{Data and Event Selection}

We used the data collected by the BABAR detector5 [4] with the total luminosity of $514 \mathrm{fb}^{-1}$. Most of the data were taken at the $\Upsilon(4 S)$ resonance plus including about $28 \mathrm{fb}^{-1}$ data at $\Upsilon(3 S)$ and $14 \mathrm{fb}^{-1}$ data at $\Upsilon(2 S)$ and $48 \mathrm{fb}^{-1}$ data at the off-resonance. The $\Upsilon(4 S)$ resonance decays to a pair of $\bar{B} B$ [5]. We used about $5 \%$ of the data set to validate and optimize the analysis method. The rest of the data was only examined after finish finalizing the analysis method. For the background study we generated signal Monte Carlo (MC) samples.

Signal MC events are generated using MadGraph 5 [6], which calculates matrix elements for the sample. The MC then were showered using Pythia 6 [7] for about 30 different $Z^{\prime}$ mass hypotheses. The main background comes from the QED processes. We generate the direct processes of $e^{+} e^{-} \rightarrow$ $\mu^{+} \mu^{-} \mu^{+} \mu^{-}$using Diag36 [8], which includes the full set of the lowest order diagrams. The Diag36 does not include initial state radiation (ISR) samples. The events of the process of $e^{+} e^{-} \rightarrow e^{+} e^{-}(\gamma)$ are generated using BHWIDE [9] and the MC events of $e^{+} e^{-} \rightarrow \mu^{+} \mu^{-}(\gamma)$ and $e^{+} e^{-} \rightarrow \tau^{+} \tau^{-}(\gamma)$ are generated using KK [10]. The off-resonance data samples, $e^{+} e^{-} \rightarrow \bar{q} q(\mathrm{q}=\mathrm{u}, \mathrm{d}, \mathrm{s}, \mathrm{c})$, are simulated using EvtGen [11]. The events processes of $e^{+} e^{-} \rightarrow \psi(2 S) \gamma$ then $\psi(2 S) \rightarrow \pi^{+} \pi^{-} J / \psi$ and $J / \psi \rightarrow \mu^{+} \mu^{-}$ were generated using a structure function technique $[12,13]$. Finally the detector acceptance and reconstruction efficiency are determined using MC simulation based on GEANT4 [14].

\section{$3 Z^{\prime}$ Measurement}

We select events containing exactly two pairs of oppositely charged tracks, consistent with the topology of the process: $e^{+} e^{-} \rightarrow \mu^{+} \mu^{-} Z^{\prime}$ and $Z^{\prime} \rightarrow \mu^{+} \mu^{-}$final state. The muons are identified by particle identification algorithms for each track. We require the sum of energies of the electromagnetic clusters that are not associated to any track must be less than $200 \mathrm{MeV}$. We finally reject events that come from the $\Upsilon(3 S)$ and $\Upsilon(2 S)$, where $\Upsilon(2 S, 3 S) \rightarrow \pi^{+} \pi^{-} \Upsilon(1 S), \Upsilon(1 S) \rightarrow \mu^{+} \mu^{-}$decays if the dimuon combination is within $100 \mathrm{MeV}$ of the $\Upsilon(1 S)$ where pions are misidentified as muons.

The distribution of the four-muon invariant mass after all selections is shown in Fig. 2 (left). At the low mass of the four-muon invariant mass, $m(4 \mu)<9 \mathrm{GeV}$, is well reproduced by the Monte Carlo simulation including direct decays of $e^{+} e^{-} \rightarrow \mu^{+} \mu^{-} \mu^{+} \mu^{-}$, however, the Monte Carlo simulation overestimates the full energy peak by $\sim 30 \%$ and fails to reproduce the radiactive tail. The overestimate simulation is expected because the Diag36 simulation does not simulate the initial state radiation (ISR) events. To estimate the potential ISR emission we select $e^{+} e^{-} \rightarrow \mu^{+} \mu^{-} \mu^{+} \mu^{-}$events by requiring a four-muon invariant mass distribution within $500 \mathrm{MeV}$ of the nominal center-of-mass energy. We also require the tracks to originate from the interaction point to within its uncertainty and constraining the center-of-mass energy of the system to be within the beam energy spread. The four-muon invariant mass after allowing the potential ISR emission is now fit as shown in Fig. 2 (right). 

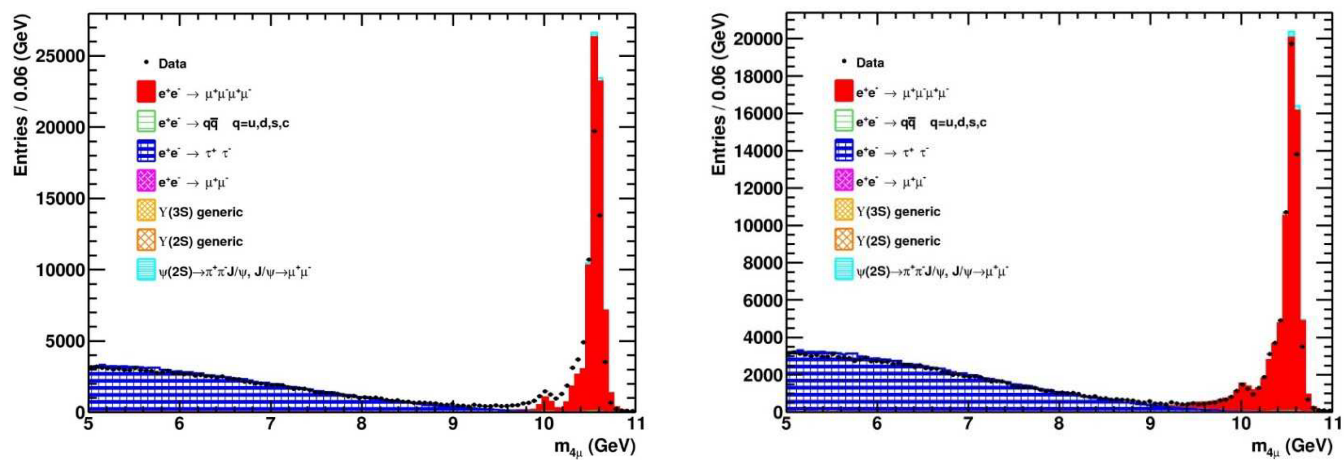

Figure 2. (Left) The four-muon invariant mass distribution with the Monte Carlo predictions of various processes including direct decay of $e^{+} e^{-} \rightarrow \mu^{+} \mu^{-} \mu^{+} \mu^{-}$normalized to the data luminosity. (Right) The four-muon invariant mass distribution with the Monte Carlo predictions of various processes by allowing the initial state radiation emission.

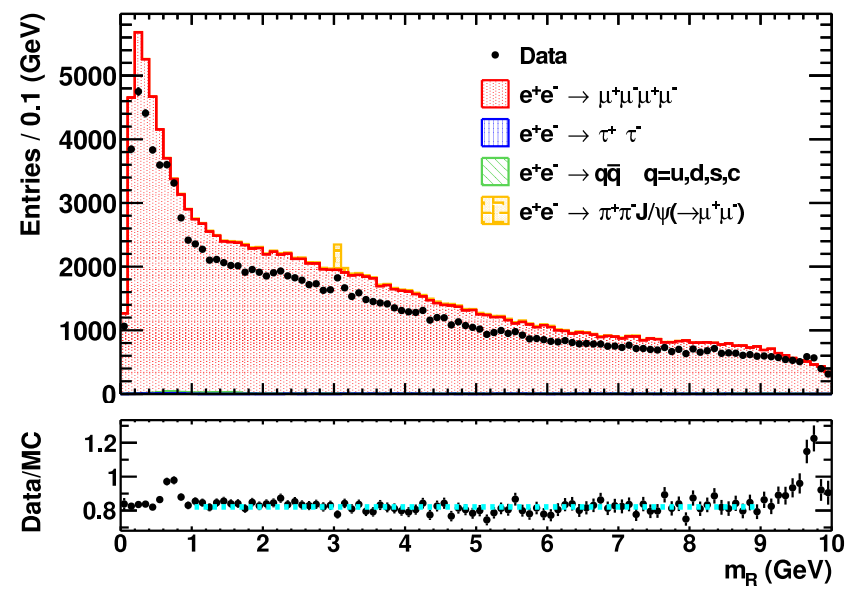

Figure 3. The distribution of the reduced dimuon mass, $m_{R}=\sqrt{m_{\mu^{+} \mu^{-}}^{2}-4 m_{\mu}^{2}}$, for the optimization sample after applying all selections, together with the Monte Carlo predictions of various processes with normalized to the data luminosity. The fit ratio between the reconstructed and simulated events is shown as a blue dashed line.

We also show the distribution of the reduced dimuon mass. The reduced dimuon mass is calculated using the following equation $m_{R}=\sqrt{m_{\mu^{+} \mu^{-}}^{2}-4 m_{\mu}^{2}}$ in linear scale as shown in Fig. 3 and in log scale as shown in Fig. 4. The most dominant samples is coming from the direct decay of $e^{+} e^{-} \rightarrow \mu^{+} \mu^{-} \mu^{+} \mu^{-}$ process. The contribution from the decay of $\Upsilon(2 S) \rightarrow \pi^{+} \pi^{-} J / \psi, J / \psi \rightarrow \mu^{+} \mu^{-}$as shown around 3 $\mathrm{GeV}$. The reduced dimoun mass distribution has a better behavior near threshold and it is also easier to model compare to the dimuon mass distribution. The signal efficiency at low masses is about $35 \%$ and it rises to about $50 \%$ around higher mass of the reduced dimuon mass. We exclude the $J / \psi$ region when calculating the correction factors by fitting the simulated and reconstructed reduced dimuon 


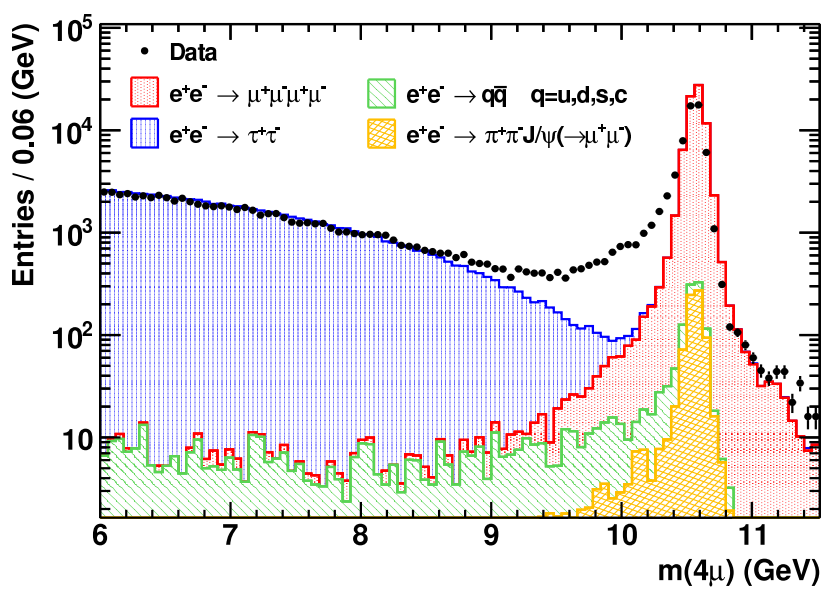

Figure 4. The distribution of the reduced dimuon mass, $m_{R}=\sqrt{m_{\mu^{+} \mu^{-}}^{2}-4 m_{\mu}^{2}}$ in log scale together with the Monte Carlo predictions of various processes.

masses in the range of $1<m_{R}<9 \mathrm{GeV}$. We obtain a correction factor of 0.82 as shown in Fig. 3 . It includes the ISR emission and differences in the trigger efficiency, charged particle identification, and track and photon reconstruction efficiencies. We assign a systematic uncertainty of $5 \%$ to cover the small variations between the uncertainties on the $e^{+} e^{-} \rightarrow \mu^{+} \mu^{-} \mu^{+} \mu^{-}$and data taking period. We calculate the ISR contribution based on the quasi real electron approximation [15]. We assess the sidebands of the four dimuon mass distribution in the range of $5.0-8.0 \mathrm{GeV}$. In this region the process of $e^{+} e^{-} \rightarrow \tau^{+} \tau^{-}(\gamma)$ is dominant. The correction factors are in agreement with the correction factors obtain from the reduce dimuon mass spectrum. The signal yield is extracted bu a series of unbinned likelihood fits to the reduced dimuon mass spectrum within the range of $0.212<m_{R}<10$ $\mathrm{GeV}$ and $0.212<m_{R}<9 \mathrm{GeV}$ for the $\Upsilon(4 S)$ resonance data and $\Upsilon(2 S)$ and $\Upsilon(3 S)$ resonances data, respectively. We exclude a region of $\pm 30 \mathrm{MeV}$ around the nominal known $J / \psi$ mass. We probe a total of 2219 mass hypothesis. The cross section of $e^{+} e^{-} \rightarrow \mu^{+} \mu^{-} Z^{\prime}, Z^{\prime} \rightarrow \mu^{+} \mu^{-}$is extracted as a function of $Z^{\prime}$ mass as shown in Fig. 5. The gray band indicates the excluded region. We find the largest local significance is $4.3 \sigma$ around $Z^{\prime}$ mass of $8.2 \mathrm{GeV}$ that is corresponding to the global significance of $1.6 \sigma$ and it is consistent with the zero-hypothesis. We also derive $90 \%$ confidence level (CL) Bayesian upper limit on the cross section of $e^{+} e^{-} \rightarrow \mu^{+} \mu^{-} Z^{\prime}, Z^{\prime} \rightarrow \mu^{+} \mu^{-}$as shown in Fig. 6.

We consider all uncertainties to be uncorrelated except for the uncertainties of the luminosity and efficiency. We finally extract the corresponding $90 \% \mathrm{CL}$ on the coupling parameter $g^{\prime}$ by assuming the equal magnitude vector couplings muons, taus and the corresponding neutrinos together with the existing limits from Borexino and neutrino experiments as shown in Fig. 7 . We set down to $7 \times 10^{-4}$ near the dimuon threshold. 


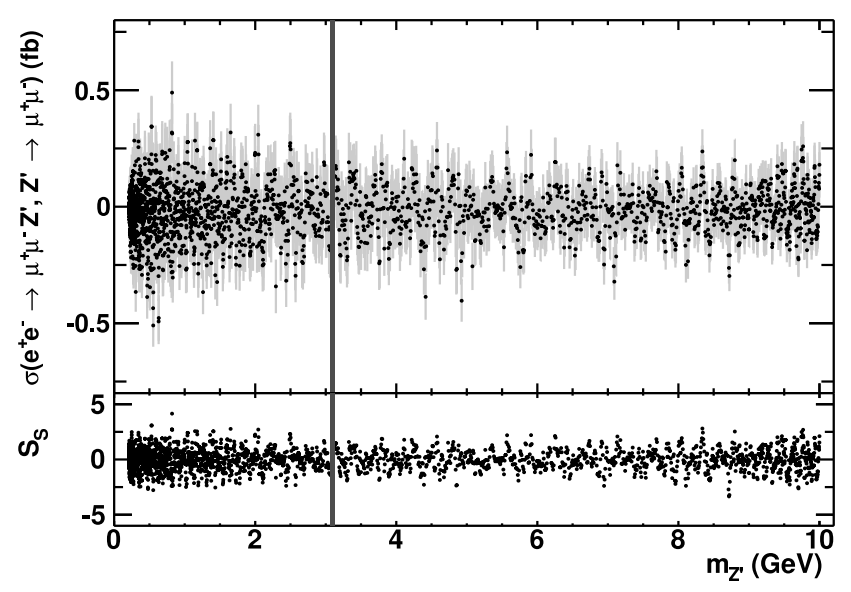

Figure 5. The measurement of $e^{+} e^{-} \rightarrow \mu^{+} \mu^{-} Z^{\prime}, Z^{\prime} \rightarrow \mu^{+} \mu^{-}$cross section with its statistical significance as a function of the $Z^{\prime}$ mass. The excluded region is indicated by the gray band.

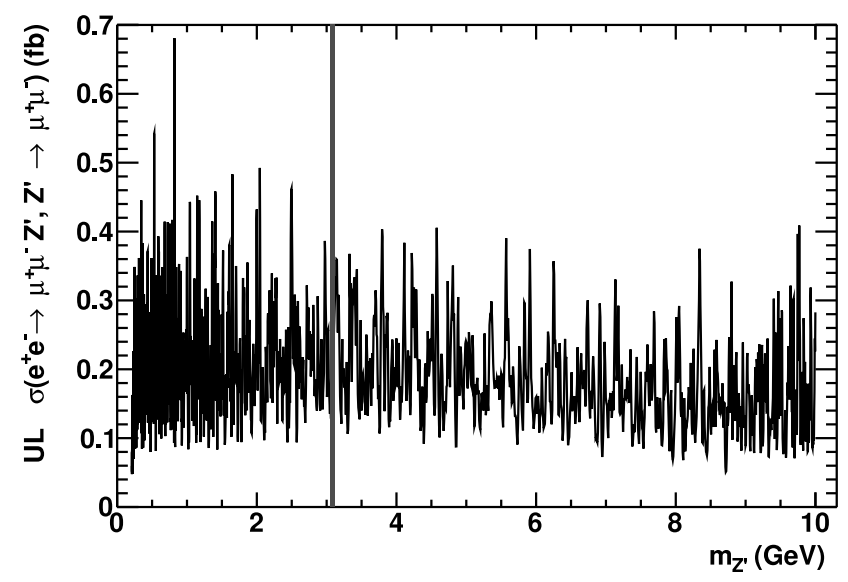

Figure 6. The limit on the cross section $\sigma\left(e^{+} e^{-} \rightarrow \mu^{+} \mu^{-} Z^{\prime}, Z^{\prime} \rightarrow \mu^{+} \mu^{-}\right)$as a function of the $Z^{\prime}$ mass. The excluded region is indicated by the gray band.

\section{Conclusion}

In conclusion, we have performed the first direct measurement of $Z^{\prime}$ production from the decay of $e^{+} e^{-} \rightarrow \mu^{+} \mu^{-} Z^{\prime}, Z^{\prime} \rightarrow \mu^{+} \mu^{-}$an $e^{+} e^{-}$collider at BABAR. No significant signal is observed for $Z^{\prime}$ masses in the range of $0.212-10 \mathrm{GeV}$. We set limits on the coupling parameters $g^{\prime}$ down to $7 \times 10^{-4}$. We set a strongest bounds for many parameter space below $3 \mathrm{GeV}$. We exclude most of the remaining parameter space preferred by the discrepancy between the calculated and measured anomalous magnetic moment of the muon above the dimuon threshold [16]. 


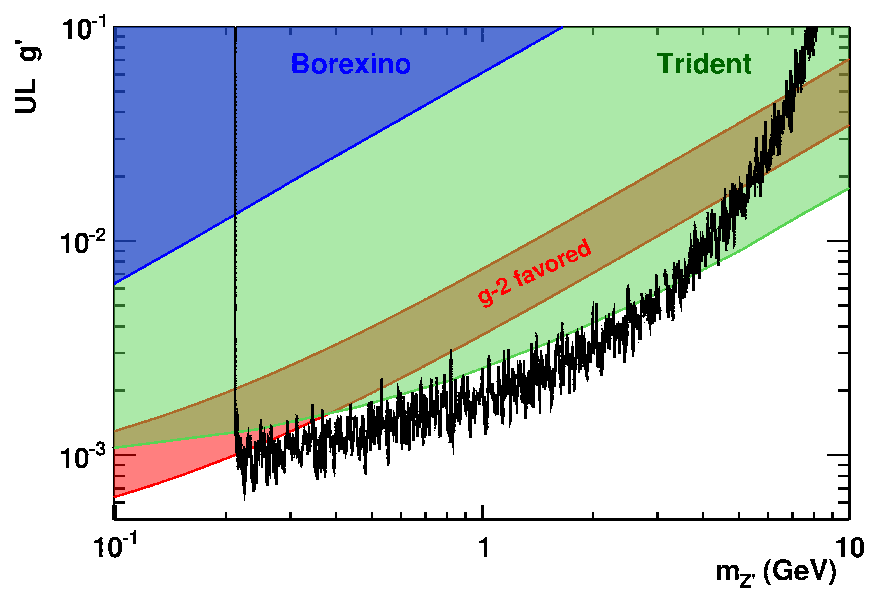

Figure 7. Upper limit on the new gauge coupling $g^{\prime}$ as a function of the mass of $Z^{\prime}$ together with the existing limits from Borexino and neutrino experiments.

\section{ACKNOWLEDGMENTS}

The author would like to thank B. Echenard for the helpful conversations. The author also thanks the organizers of the XLVI International Symposium on Multiparticle Dynamics, Jeju Island, South Korea. The support from the BABAR Collaboration, the University of South Alabama, and the University of Mississippi is gratefully acknowledged. This work was partially supported by the U.S. Department of Energy.

\section{References}

[1] A. Birkedal, K. Matchev, and M. Perelstein, Phys. Rev. D 70, 077701 (2004)

[2] B. Holdom, Phys. Lett. B 166, 196 (1986)

[3] W. Altmannshofer, S. Gori, M. Pospelov, and I. Yavin Phys. Rev. D 89, 095033 (2014)

[4] B. Aubert et al., (BABAR Collaboration), Nucl. Instrum. Meth. A 729, 1 (2013)

[5] B. Aubert et al., (BABAR Collaboration), Phys. Rev. Lett. 95, 042001 (2005)

[6] J. Alwall et al., JHEP 1407, 079 (2014)

[7] T. Sjostrand, S. Mrenna, and P. Z. Skands, JHEP 0605, 026 (2006)

[8] F. A. Berends, P. H. Daverveld and R. Kleiss, Nucl. Phys. B 253, 441 (1985)

[9] S. Jadach, W. Placzek, and B. F. L. Ward, Phys. Lett. B 390, 298 (1997)

[10] S. Jadach, B. F. L. Ward, and Z. Was, Phys. Rev. D 63, 113009 (2001)

[11] D. J. Lange, Nucl. Instrum. Meth. A 462, 152 (2001)

[12] A. B. Arbuzov et al., J. High Energy Phys. 9710, 001 (1997)

[13] M. Caffo, H. Czyz, and E. Remiddi, Nuovo Cim. A110 515 (1997); Phys. Lett. B327, 369 (1994)

[14] S. Agostinelli et al., (GEANT4 Collaboration), Nucl. Instrum. Meth. A 506, 250 (2003)

[15] M. Benayoun, S. I. Eidelman, V. N. Ivanchenko and Z. K. Silagadze, Mod. Phys. Lett. A 14, 2605 (1999)

[16] J. P. Lees et al., (BABAR Collaboration), Phys. Rev. D 94, 011102 (2016) 